\title{
ВMJ Global Health Understanding pretreatment loss to follow-up of tuberculosis patients: an explanatory qualitative study in Chennai, India
}

\author{
Beena E Thomas, ${ }^{1}$ Chandra Suresh, ${ }^{1} \mathrm{~J}$ Lavanya, ${ }^{2}$ Mika M Lindsley, ${ }^{3}$ \\ Amith T Galivanche, ${ }^{3}$ Senthil Sellappan, ${ }^{1}$ Senthanro Ovung, ${ }^{1}$ Amritha Aravind, ${ }^{1}$ \\ Savari Lincy, ${ }^{1}$ Agnes Lawrence Raja, ${ }^{1}$ S Kokila, ${ }^{1}$ B Javeed, ${ }^{1}$ S Arumugam, ${ }^{1}$ \\ Kenneth H Mayer, ${ }^{4,5}$ Soumya Swaminathan, ${ }^{6}$ Ramnath Subbaraman (D) ${ }^{3,7}$
}

To cite: Thomas BE,

Suresh C, Lavanya J, et al. Understanding pretreatment loss to follow-up of tuberculosis patients: an explanatory qualitative study in Chennai, India. BMJ Global Health 2020;5:e001974. doi:10.1136/ bmjgh-2019-001974

Handling editor Stephanie M Topp

- Additional material is published online only. To view please visit the journal online (http://dx.doi.org/10.1136/ bmjgh-2019-001974)

BET and RS contributed equally.

Received 9 September 2019 Revised 18 December 2019 Accepted 13 January 2020

Check for updates

(C) Author(s) (or their employer(s)) 2020. Re-use permitted under CC BY-NC. No commercial re-use. See rights and permissions. Published by BMJ.

For numbered affiliations see end of article.

Correspondence to Dr Ramnath Subbaraman; ramnath.subbaraman@tufts.edu

\section{ABSTRACT}

Introduction Pretreatment loss to follow-up (PTLFU)dropout of patients after diagnosis but before treatment registration-is a major gap in tuberculosis (TB) care in India and globally. Patient and healthcare worker (HCW) perspectives are critical for developing interventions to reduce PTLFU.

Methods We tracked smear-positive TB patients diagnosed via sputum microscopy from 22 diagnostic centres in Chennai, one of India's largest cities. Patients who did not start therapy within 14 days, or who died or were lost to follow-up before official treatment registration, were classified as PTLFU cases. We conducted qualitative interviews with trackable patients, or family members of patients who had died. We conducted focus group discussions (FGDs) with HCWs involved in TB care. Interview and FGD transcripts were coded and analysed with Dedoose software to identify key themes. We created categories into which themes clustered and identified relationships among thematic categories to develop an explanatory model for PTLFU.

Results We conducted six FGDs comprising 53 HCWs and 33 individual patient or family member interviews. Themes clustered into five categories. Examining relationships among categories revealed two pathways leading to PTLFU as part of an explanatory model. In the first pathway, administrative and organisational health system barriers-including the complexity of navigating the system, healthcare worker absenteeism and infrastructure failures-resulted in patients feeling frustration or resignation, leading to disengagement from care. In turn, HCWs faced work constraints that contributed to many of these health system barriers for patients. In the second pathway, negative HCW attitudes and behaviours contributed to patients distrusting the health system, resulting in refusal of care.

Conclusion Health system barriers contribute to PTLFU directly and by amplifying patient-related challenges to engaging in care. Interventions should focus on removing administrative hurdles patients face in the health system, improving quality of the $\mathrm{HCW}$-patient interaction and alleviating constraints preventing HCWs from providing patient-centred care.

\section{Key questions}

What is already known?

- Pretreatment loss to follow-up (PTLFU) is a key gap in tuberculosis (TB) care delivery globally and in India, where estimates suggest that $>200000 \mathrm{pa}$ tients experience PTLFU annually in the country's national TB program.

- Prior Indian studies have mostly focussed on understanding patient-related challenges-rather than health system barriers-contributing to PTLFU.

\section{What are the new findings?}

- Administrative and organisational barriers in the health system-including the complexity of navigating the system and healthcare worker absenteeism-resulted in some TB patients feeling frustration or resignation, leading to disengagement from care.

- Negative or judgemental healthcare worker attitudes and behaviours contributed to some patients distrusting the health system, resulting in refusal of further care.

- Patients-related challenges-including presentation with advanced illness, alcohol use disorder, work constraints and poverty—also contributed to PTLFU.

What do the new findings imply?

- Health system barriers play a central role in contributing to PTLFU.

- Removing administrative hurdles by alleviating constraints faced by healthcare workers, and improving quality of the healthcare worker-patient interaction may help to reduce PTLFU.

\section{INTRODUCTION}

Tuberculosis (TB) care may be undermined by patient loss to follow-up across sequential steps from care seeking to successful treatment, comprising the care cascade. ${ }^{1}$ Pretreatment loss to follow-up (PTLFU), which refers to patient losses after diagnosis but before treatment registration, is a key gap in the care 
cascade in several high TB burden countries. ${ }^{23}$ An analysis conducted in India, which accounts for one-fourth of cases and one-third of TB deaths globally, estimated that $>200000$ patients experience PTLFU annually in the national TB programme. ${ }^{4}$

Prior Indian studies of PTLFU have tracked down 'lost' patients to obtain their perspectives on why they dropped out of care. While providing valuable information, these studies have generally reported lists of reasons patients did not start treatment, without analysing their narrative responses or producing a framework to inform interventions. Most studies assumed that PTLFU results from a patient decision not to seek further care, rather than from more complex interactions between patients and the health system. As such, these studies have mostly reported patient factors for PTLFU, including presentation with advanced illness, ${ }^{56}$ low literacy, ${ }^{7}$ work constraints, ${ }^{689}$ alcohol use, ${ }^{8}$ denial of diagnosis, ${ }^{7}$ urbanrural movement ${ }^{6-11}$ and not collecting sputum test results. ${ }^{57}$ When considered at all, health system barriers emerged indirectly, including patient dissatisfaction with health services, ${ }^{5-8}$ concerns about being monitored via facility-based directly observed therapy (DOT) ${ }^{57}$ or inability to find patients due to poor recording of their contact information. ${ }^{6712}$

In this paper, we analyse qualitative data on PTLFU from TB patients and healthcare workers (HCWs) in Chennai, one of India's largest cities. Data collection was embedded within a cohort study in Chennai's government TB programme, which found that more than onefifth of smear-positive patients experienced PTLFU. ${ }^{13}$ The cohort study showed that patients with a prior TB treatment history were at higher risk for PTLFU, which is concerning because these patients are more likely to have drug-resistant $\mathrm{TB}$, such that failure to start treatment could contribute to transmission of such strains.

Although this previous quantitative study identified who is more likely to experience PTLFU, it did not shed light on why patients with a life-threatening disease did not officially register in treatment. The current qualitative study provides insights into PTLFU that cannot be gleaned using quantitative methods alone. In contrast to prior Indian studies, we also propose an explanatory model that views PTLFU as resulting from a dynamic interaction between patients and the health system. The goal of this model is to inform intervention development to reduce PTLFU in urban India.

\section{METHODS \\ Study setting}

Chennai has a population of 7.1 million people and a high TB prevalence of about 349 per 100000 people. ${ }^{14}$ City TB services follow guidelines of India's Revised National TB Control Programme (RNTCP). The current study followed patients at 22 of Chennai's RNTCP-designated microscopy centres (DMCs), which accounted for $90 \%$ of smear-positive TB patients diagnosed in the public sector in 2014. ${ }^{13}{ }^{15}$ Four DMCs, located in specialised TB facilities or tertiary hospitals, diagnosed more than half of the city's smear-positive patients; ${ }^{15}$ we refer to these four as 'high-volume DMCs' and to the remaining 18 as 'moderate-volume or low-volume DMCs'.

\section{Case definitions for pretreatment loss to follow-up}

We discuss case definitions briefly, as they have been described in detail in the prior quantitative manuscript. ${ }^{13}$ Patients diagnosed with smear-positive TB via sputum microscopy had to traverse a multistep process of diagnosis and linkage to care. Depending on whether they were initially managed as outpatients or inpatients, this multistep process differed, but both groups were included in our sample. Patients were lost at three stages (figure 1): during diagnostic workup, during outpatient referral or hospital admission or during the official RNTCP registration process because registration was delayed for

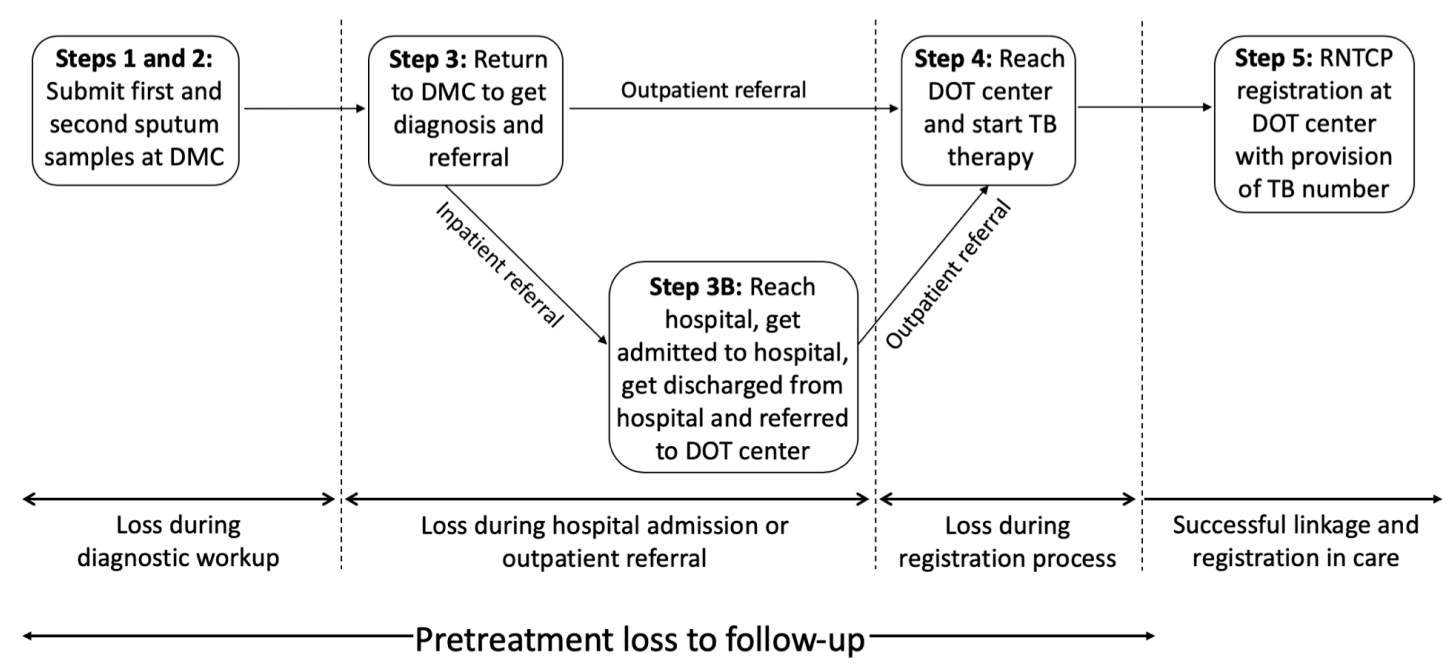

Figure 1 Multistep process of diagnosis and linkage to care for smear-positive TB patients diagnosed by sputum microscopy in Chennai, India. Modified from Thomas et al 2018. ${ }^{13}$ DMC, designated microscopy centre; DOT, directly observed therapy; RNTCP, Revised National TB Control Programme; TB, tuberculosis. 
more than 2 weeks for nearly one-third of patients. ${ }^{13}$ Patients who completed all stages were considered to have successfully linked to care. Conversely, we defined PTLFU as including two types of patients: (1) those who did not start therapy in the public sector within 14 days of initial sputum evaluation, due to loss to follow-up, death or delay; or (2) those who started therapy but were lost to follow-up or died before official registration. ${ }^{13}$ For this qualitative study, our PTLFU patient sample therefore included individuals who dropped out at different points in this multistep process, providing insights into diverse challenges involved in linking to care.

\section{Field methods for evaluating pretreatment loss to follow-up}

Between October 2015 and June 2016, we followed all patients $\geq 18$ years old diagnosed with smear-positive TB by sputum microscopy during a 4 -week time period at each of the 22 DMCs. ${ }^{13}$ Patients were tracked starting no earlier than 14 and no later than 21 days after their first positive sputum test, using a systematic process involving health record audits, phone outreach and home visits if necessary. ${ }^{13}$ We continued to follow patients who started treatment within 14 days until they were officially registered (ie, given a TB Number). We classified patients as being untrackable if we were unable to find them after multiple phone calls and at least one home visit.

\section{Collection of qualitative patient data}

We collected qualitative interviews between October 2015 and June 2016, concordant with the cohort study. Interviews lasted 30 to $45 \mathrm{~min}$ and were conducted by researchers with a master's degree in social work. After obtaining informed consent, interviews were audio-recorded in Tamil and translated to produce English transcripts.

Out of 76 PTLFU patients in the cohort, 27 were untrackable and could not be interviewed. ${ }^{13}$ We interviewed 20 out of 28 PTLFU patients who were alive and trackable by our team. Six patients declined to be interviewed, one patient from Andhra Pradesh was unable to be interviewed due to travel considerations and the audio recording for one interview could not be analysed because it was muffled. We also interviewed family members of 13 out of 21 PTLFU patients who died before official registration. As such, we report findings for $\mathrm{n}=33$ PTLFU patients. Notably, only three women were included in the sample because women were more likely than men to have declined an interview, to have been untrackable, and to have not had family members interviewed by our team if they had died.

We used interview guides to elicit narrative information on the event timelines and challenges leading to PTLFU. Questions varied based on when a patient was 'lost' (ie, during diagnosis, outpatient referral, hospital admission or registration). For example, for patients who did not reach a DOT centre for treatment, a key question was 'What were reasons you were not able to make it to the (DOT) centre to start treatment?' Language was modified as needed for interviews with patients' family members.

\section{Collection of qualitative healthcare worker data}

Between July 2016 and December 2016, we conducted two focus group discussions (FGDs) each with three types of health personnel (six FGDs total) in Chennai's TB programme: (1) Laboratory Technicians, who collect sputum samples, record patients' contact information and evaluate sputum for evidence of acid-fast bacilli; (2) Health Visitors, who counsel and refer patients (at DMCs) or start them on treatment (at DOT centres) and (3) Senior Treatment Supervisors, who supervise Health Visitors.

FGDs were conducted after monthly meetings of HCWs from across Chennai at the district TB office. As such, FGDs included a relatively representative sample of HCWs from geographically diverse areas of the city and all levels of health facilities (primary, secondary and tertiary centres). FGDs lasted 45 to $60 \mathrm{~min}$ and were conducted in Tamil by researchers with social work backgrounds. FGD guides included open-ended questions to elicit feedback on reasons for PTLFU.

\section{Data analysis}

We conducted a thematic analysis of interviews and FGDs using an inductive approach. ${ }^{16}{ }^{17}$ We created an initial coding scheme-using 'health system barriers' and 'patient-related, family-related and society-related barriers' as two preliminary working categories-based on discussion within the research team, including principal investigators (BET, RS) and field investigators who collected the data (SL, ALR, SK, BJ, SS and CS). Patient interviews were independently coded a first time by three researchers (RS, SO, AA) using Dedoose software (V.8.0.35, Los Angeles, California: SocioCultural Research Consultants, LLC). Researchers met frequently to reconcile differences in code application and identify new themes emerging from the data. After incorporating new themes, all interviews were coded a second time (by RS, ML, SO and AA) using the revised scheme (online supplementary figure S1). A similar process of constructing an initial coding scheme, coding all interviews, revising the coding scheme with emergent themes and then recoding all data was performed for HCW FGDs (by AG, ML and RS) (online supplementary figure S2).

We then conducted a deeper analysis with the goal of identifying interconnections among themes in the patient and HCW data to develop a broader explanatory model for PTLFU. This approach was inspired by previous studies that used meta-ethnography ${ }^{18}$ and thematic synthesis ${ }^{19}$ to develop explanatory models for patient non-engagement in HIV or TB care. ${ }^{20-23}$ Using Dedoose, we re-evaluated common or salient themes from our initial coding to ascertain larger categories into which themes clustered.

Given the health system's dominant role in patient and HCW narratives, we first separated out into one category patient-related, family-related or society-related challenges contributing to PTLFU, with a key criterion being that patients' interactions with the health system were unrelated or only indirectly related to these themes. 
Other categories were created by analysing remaining themes from patient and HCW data that focussed on the health system's role. One category emerged from the observation that numerous themes in the patient interviews related to administrative or organisational barriers in the health system. Another category emerging from the patient interviews reflected negative patient attitudes and behaviours towards the health system that were driven by strong emotions, such as distrust or fear. In the FGDs, HCWs described diverse constraints they faced when trying to deliver care, and we compiled those themes into another category. The last category, which compiled themes on negative HCW attitudes and behaviours towards patients, was the most complex because it relied on descriptions of HCW actions provided by both patients and HCWs. While we place greater weight on patient descriptions of negative HCW actions, we also believe that HCWs' own reports on this issue are relevant. We assume that, because of social desirability, HCWs would be biassed towards presenting more positive perspectives on the health system. As such, we found HCWs' descriptions of negative or counter-productive behaviours towards patients, performed by themselves or other HCWs, to be forms of self-reflection and selfcritique that provided valuable insights.

Finally, to inform the explanatory model, we conducted a mapping exercise in which we tracked logical interconnections among thematic categories using specific themes or representative quotations. For example, several HCWs noted that having to work at multiple facilities was a constraint in care delivery (in thematic category 4), and this finding connected with patients' descriptions of not being able to get test results or referrals due to HCW absenteeism (in thematic category 2). After mapping several such interconnections, we assembled these findings into our explanatory model.

For representative quotations, we report study participants' descriptive characteristics, such as job title for HCWs and sex and prior TB treatment history for patients. In doing so, we do not mean to imply that thematic saturation has been achieved for specific subgroups; rather, these characteristics are intended to provide readers with the general background of the patient or HCW from whom the quotation was drawn.

\section{Patient and public involvement}

Feedback on the study protocol was provided by the National Institute for Research in TB's Community Advisory Board, which includes TB advocates and former TB patients.

\section{RESULTS}

\section{Descriptive characteristics of study participants}

Of 33 TB patient interviews, 13 (39\%) were conducted with family members of patients who had died. Patients' ages ranged from 21 to 80 (median 53) years. Of the sample, $3(9 \%)$ were women, $4(12 \%)$ lived in rural areas outside Chennai, 25 (76\%) were diagnosed at highvolume DMCs and $21(64 \%)$ had a prior TB treatment history.

The six FGDs included 53 participants, of whom 18 (34\%) were Senior Treatment Supervisors, 18 (34\%) were Health Visitors and 17 (32\%) were Laboratory Technicians. Sex and years of RNTCP work experience were captured for 40 participants (ie, four FGDs), of whom 30 $(75 \%)$ were men. Work experience ranged from 1 to 30 (median 5.5) years.

\section{Thematic categories}

Barriers contributing to PTLFU clustered into five thematic categories. Three categories emerged from patient or family member interviews and reflected patient experiences, attitudes or behaviours: (1) patientrelated, family-related and society-related challenges contributing to PLTFU that are largely independent of the health system, (2) health system organisational and administrative barriers experienced by patients and (3) patient attitudes and behaviours towards the health system contributing to PTLFU. The remaining two categories mostly emerged from the FGDs and reflected HCW experiences, attitudes or behaviours: (4) social and resource constraints in the health system that limit HCWs' ability to do their jobs effectively and (5) HCW attitudes and behaviours towards patients contributing to PTLFU.

Thematic category 1: patient-related, family-related and societyrelated challenges that are largely independent of the health system

Some themes contributing to PTLFU reflected patient challenges at the individual or societal levels that were unrelated to interactions with the health system (table 1). At the individual level, presentation with advanced illness from TB (Q1) and comorbidities such as alcohol use disorder (Q2) and depression (Q3) made it difficult for patients to navigate health facilities and contributed to deaths before official registration. One family member described how depression led to PTLFU and death after the patient was initially hospitalised for advanced illness:

(After being admitted to the hospital,) my mother said to me, 'If you don't take me home, I will cut my throat'... they discharged us at 2 p.m. (Family member of a woman with a prior treatment history).

At the family level, patients sometimes did not follow-up sputum test results because they were attending family functions or festivals in villages outside of Chennai (Q4). Some patients experienced TB-related stigma within their families or lacked support to be accompanied to medical visits (Q5, Q6), two barriers which sometimes intersected:

I came here without my family members' knowledge, as they otherwise would not allow me to visit (this TB speciality hospital). If others learn that I am visiting (this hospital), 
Table 1 Representative quotations on patient-related, family-related and society-related challenges

\begin{tabular}{|c|c|}
\hline Challenge & Representative quotation \\
\hline $\begin{array}{l}\text { Presentation with } \\
\text { advanced illness }\end{array}$ & $\begin{array}{l}\text { Q1. Even at the time of admission (to the hospital), the doctor said that his illness was too advanced. } \\
\text { So the doctor advised me to take care of him at home... Three days after talking to the doctor my } \\
\text { father died. (Family member of a man with a prior treatment history). }\end{array}$ \\
\hline Alcohol use disorder & $\begin{array}{l}\text { Q2. He consumed alcohol. For that reason, he didn't take TB medications properly. (Family member of } \\
\text { a man with a prior treatment history). }\end{array}$ \\
\hline Depression & $\begin{array}{l}\text { Q3. I stopped my treatment due to depression, because I came to know I had TB. (Man with a prior } \\
\text { treatment history) }\end{array}$ \\
\hline $\begin{array}{l}\text { Attending family } \\
\text { function or festival }\end{array}$ & $\begin{array}{l}\text { Q4. I went back to my native village for (my grandfather's) funeral rites. I had to take care of all } \\
\text { formalities... I couldn't go back to pick up my TB test report on time. (Man without a prior treatment } \\
\text { history) }\end{array}$ \\
\hline $\begin{array}{l}\text { Lack of social support } \\
\text { from family members }\end{array}$ & $\begin{array}{l}\text { Q5. No one is there to take care of me; even my children are ignoring me. (Man with a prior treatment } \\
\text { history) }\end{array}$ \\
\hline Poverty & $\begin{array}{l}\text { Q8. I did not bring my brother immediately (to the hospital) because we don't have the money. (Family } \\
\text { member of a man with a prior treatment history) }\end{array}$ \\
\hline Homelessness & $\begin{array}{l}\text { Q9. We don't have a house; we lived on the platform (Indian term for homelessness) for } 9 \text { months. } \\
\text { (Family member of a man without a prior treatment history) }\end{array}$ \\
\hline
\end{tabular}

TB, tuberculosis.

they will tag me as being a TB patient. (Woman without a prior treatment history)

At the societal level, work constraints $\left(Q^{7}\right)$, poverty (Q8), homelessness (Q9) and weather-related constraints contributed to PTLFU. For example, the following patient could not get permission to leave work and could not afford to miss a single day's wages due to poverty:

I got my (sputum test) report only after 1 week due to work. It was very difficult to get leave approval from my office, and my family depends on my salary, as I am getting daily wages. (Man with a prior treatment history).

Thematic category 2: organisational and administrative barriers in the health system experienced by patients

Thematic category 2 comprises organisational and administrative barriers that made navigating the health system an intimidating and confusing experience (table 2). Patients described difficulties finding outpatient clinics within tertiary hospitals (Q10), having to return to the same health facility repeatedly (Q11) and having to navigate between facilities for diagnosis and treatment initiation (Q12, Q13). The family member of one patient who died described frustration in traversing a tertiary hospital when the patient was ill:

We went to (a tertiary hospital) for initial check-up... They didn't tell us much. They said go to number 3 (outpatient clinic) and then number 5 (outpatient clinic) and back again for 2 days. After running from pillar to post, we just gave up and returned home. (Family member of a man without a prior treatment history).
Navigational challenges were compounded by other administrative problems. Patients sometimes had low motivation to pursue next steps in care because HCWs did not provide general TB information (Q14) or inform them of their diagnoses, as described by the following patient:

No one told me about my TB diagnosis. (Woman without a prior treatment history).

Even when they navigated to the right location, further progress along the care pathway was often impeded by HCW demands for referral documents from diagnostic facilities (Q15), infrastructure failures (eg, of computers or electricity) (Q16) or HCW absenteeism (Q17). The following patient traversed referrals across multiple facilities, only to find that the appropriate HCW was not available:

After diagnosis, they referred me to (a local primary health centre)... the HCW referred me from there to (a second primary health centre)... The next day I went to that (second primary health centre)... However, the hospital staff asked me to return the next day, because the responsible HCW was not available. (Man with a prior treatment history).

Finally, previous experiences with facility-based DOTthe main treatment monitoring approach at the time of this study-deterred some patients with a prior TB history from engaging in further care. These patients found frequent visits to DOT centres to be incompatible with work and life demands (Q18). 
Table 2 Representative quotations on organisational and administrative barriers in the health system experienced by patients

\begin{tabular}{|c|c|}
\hline Health system barrier & Representative quotation \\
\hline $\begin{array}{l}\text { Complexity of navigation within } \\
\text { tertiary facilities }\end{array}$ & $\begin{array}{l}\text { Q10. For all tests, the patient has to wander here and there; otherwise they won't collect } \\
\text { the test. (Family member of a man with a prior treatment history). }\end{array}$ \\
\hline $\begin{array}{l}\text { Challenges of repeatedly returning to } \\
\text { the same facility }\end{array}$ & $\begin{array}{l}\text { Q11. Many patients are not able to come to the hospital several times, especially old } \\
\text { people. It will be very helpful if the number of required visits is reduced. (Woman without } \\
\text { a prior treatment history). }\end{array}$ \\
\hline \multirow[t]{2}{*}{$\begin{array}{l}\text { Complexity of navigation between } \\
\text { facilities }\end{array}$} & $\begin{array}{l}\text { Q12. (The HCW) told me to go to Old General Hospital. I thought that by 'Old General } \\
\text { Hospital' she meant Madras Medical College, which is very far from where I live. So I } \\
\text { went back home... I later found out that Old General Hospital meant (a local hospital), } \\
\text { which is nearby, just opposite this clinic. (Man without a prior treatment history). }\end{array}$ \\
\hline & $\begin{array}{l}\text { Q13. Yes, madam, I visited (a TB speciality hospital); they referred me to (a secondary } \\
\text { hospital) and then they referred me to (a tertiary hospital). (Man without a prior treatment } \\
\text { history). }\end{array}$ \\
\hline $\begin{array}{l}\text { Failure to notify patient of diagnosis } \\
\text { or to provide general TB information }\end{array}$ & $\begin{array}{l}\text { Q14. They referred me to a hospital for treatment... But they did not say anything about } \\
\text { my health condition and my disease. (Man without a prior treatment history). }\end{array}$ \\
\hline $\begin{array}{l}\text { Healthcare worker demanded } \\
\text { documents }\end{array}$ & $\begin{array}{l}\text { Q15. (After the patient reached the rural DOT centre, the HCW said): "Go back to } \\
\text { (tertiary hospital where the patient was diagnosed in the city) and bring a referral slip- } \\
\text { only then can we start treatment." (Man without a prior treatment history). }\end{array}$ \\
\hline Infrastructure failures & $\begin{array}{l}\text { Q16. Thrice I came to (a tertiary hospital) to receive my test report but... (t)hey said, "... } \\
\text { (Y)ou have to wait for some days... We can prepare your report only when the power } \\
\text { supply is available." (Woman without a prior treatment history). }\end{array}$ \\
\hline Healthcare worker absenteeism & $\begin{array}{l}\text { Q17. The X-ray technician said that he... can only give the result tomorrow. I pleaded } \\
\text { with him. I told him I felt too ill. He asked me to come after } 1 \text { hour... At that time the } \\
\text { doctor had left the hospital. The Sister (nurse) asked me to come back on Wednesday. } \\
\text { (Man with a prior treatment history). }\end{array}$ \\
\hline Rigidity of facility-based DOT & $\begin{array}{l}\text { Q18. (HCWs who perform DOT) come at } 11 \text { a.m. Patients will be standing in the queue; } \\
\text { they call us one by one to give the tablets. We need to take tablets in front of them } \\
\text { and sign. It almost takes until } 12 \text { p.m. Then how can I go to work? (Man with a prior } \\
\text { treatment history). }\end{array}$ \\
\hline
\end{tabular}

DOT, directly observed therapy; HCW, healthcare worker; TB, tuberculosis.

Thematic category 3: negative patient attitudes and behaviours towards the health system

Some patients or family members expressed negative emotions or attitudes towards the health system. These attitudes were more common in patients with a prior treatment history and often reflected previous or current negative experiences with government services (table 3). These included distrust (Q19, Q20), fear of medication toxicities $(\mathrm{Q} 21)$ and fear of medical care more generally (Q22, Q23). For example, one patient described how he was quick to doubt his doctor's competence:

I got worried when I heard the doctor asking the nurse whether he should be prescribing a particular tablet. (Man with a prior treatment history).

Such attitudes negatively affected care-seeking behaviour. While patients were referred often across health facilities by HCWs, in some cases, patients themselves sought care at multiple facilities without referral:

I didn't get proper treatment at (the first tertiary hospital) due to lack of staff, and I left the (second tertiary hospital) due to lack of hygiene and cleanliness. So I decided to go to (a third facility) for further care. (Man without a prior treatment history).
Care seeking at multiple sites resulted in disengagement from health facilities before patients could pick up test results or get referred for treatment. The term 'doctor shopping' is sometimes used to describe this behaviour in India; however, this term implies patient choice. In contrast, patients usually sought care at multiple sites because of concerns about quality of care or hygiene at prior facilities (Q24). As we will discuss in detail later, refusal of further care was a more concerning behaviour resulting from these patient attitudes (Q21 to Q23, Q25).

Thematic category 4: social and resource constraints that limit the ability of HCWs to effectively do their jobs

HCWs reported constraints that limited their ability to effectively perform their jobs (table 4). All three types of HCWs reported having to work at multiple facilities (Q26), which was problematic for those who were supposed to follow-up with patients on a daily basis during treatment initiation (Health Visitors) or diagnostic workup (Laboratory Technicians):

We need manpower... (O)ne Laboratory Technician is working at four DMCs. If I collect the first sputum of the patient today (Friday), I will get a chance to collect the 
Table 3 Representative quotations on patient attitudes and behaviours towards the health system

\begin{tabular}{ll}
\hline Patient attitude or behaviour & Representative quotation \\
\hline Distrust of the health system & $\begin{array}{l}\text { Q19. I know about (the nearby government hospital). I don't like that place. So I came to } \\
\text { (a tertiary hospital). In (the tertiary hospital), they maintain cleanliness. (Man with a prior } \\
\text { treatment history). } \\
\text { Q20. I don't think they (government clinics) provide good care. (Man with a prior treatment } \\
\text { history). }\end{array}$ \\
$\begin{array}{ll}\text { Fear of medication toxicities } \\
\text { Fear of further medical care } \\
\text { tablets, I will feel very giddy.' (Family member of a man with a prior treatment history) }\end{array}$ \\
$\begin{array}{l}\text { Q22. Yes, the doctor admitted him in the ward... (T)wo patients died close to my husband... } \\
\text { the next night two more patients also died there so my husband became very scared and we } \\
\text { discharged him. (Family member of a man without a prior treatment history). } \\
\text { Q23. (H)is mind didn't accept the fact that he has to take treatment for } 6 \text { months. (Family } \\
\text { member of a man with a prior treatment history) }\end{array}$ \\
$\begin{array}{l}\text { Q24. We went to (a TB outpatient clinic) where he was diagnosed... Whenever he took tablets } \\
\text { he felt drowsy, so we decided to try treatment (at a TB speciality hospital)... but he left there } \\
\text { so we took him to (another TB speciality hospital). (Family member of a man with a prior } \\
\text { treatment history). } \\
\text { Q25. When they gave him tablets, he would keep them in his hands and throw them away } \\
\text { later. That is what happened at (the inpatient hospital) ... After a few days, he ran away from } \\
\text { the hospital. (Family member of a man with a prior treatment history). }\end{array}$ \\
\hline Refusal of further care
\end{tabular}

TB, tuberculosis.

second sputum of that patient only on the next Friday.

(Participant in a Laboratory Technician FGD)

Absence of RNTCP staff contributed to poor recording of patient contact information, because non-RNTCP staff were not sure how to record this information or did not understand its importance (Q27). At high-volume facilities, HCWs had little time to spend accurately recording information (Q28).

HCWs were unable to complete routine tasks, including counselling, due to hierarchy in the health

Table 4 Representative quotations on social and resource constraints that limit HCWs' ability to effectively do their jobs

\section{Constraint on healthcare workers Representative quotation}

\begin{tabular}{|c|c|}
\hline Having to work at multiple facilities & $\begin{array}{l}\text { Q26. Health visitor 1: We are covering more than one (facility) madam. } \\
\text { Health visitor 1: Four centres madam. } \\
\text { Health visitor 2: Two centres. } \\
\text { Health visitor 6: Six centres madam. } \\
\text { Health visitor 9: For this reason, we aren't able to treat patients well. (Excerpt from a } \\
\text { health visitor FGD) }\end{array}$ \\
\hline & $\begin{array}{l}\text { Q27. General staff do not collect patient details properly. We (RNTCP staff) know that, } \\
\text { if information is not collected properly, in the future the patient may become an initial } \\
\text { defaulter (ie, PTLFU case). So we give great importance to recording patient details, but } \\
\text { other staff do not. (Participant in a laboratory technician FGD) }\end{array}$ \\
\hline $\begin{array}{l}\text { High patient volume at certain } \\
\text { facilities }\end{array}$ & $\begin{array}{l}\text { Q28. It is very difficult (to verify patient contact information) in big centres (ie, high- } \\
\text { volume facilities) because they are regularly overcrowded with patients. (Participant in a } \\
\text { senior treatment supervisor FGD) }\end{array}$ \\
\hline Hierarchy in the health system & $\begin{array}{l}\text { Q29. They call on me while l'm trying to do my work... They send someone to get me } \\
\text { saying, 'Call that RNTCP girl.' (Participant in a health visitor FGD). }\end{array}$ \\
\hline $\begin{array}{l}\text { Lack of material support to track } \\
\text { patients }\end{array}$ & $\begin{array}{l}\text { Q30. Most villages do not have any buses... those of us who have two-wheelers make } \\
\text { these visits (to track PTLFU cases), but we are not receiving any reimbursement for } \\
\text { petrol costs. (Participant in a health visitor FGD) }\end{array}$ \\
\hline $\begin{array}{l}\text { Lack of material support to } \\
\text { coordinate patient referrals }\end{array}$ & $\begin{array}{l}\text { Q31. Due to practical difficulties we never send this column (copy of the referral form). } \\
\text { If I have to send this column (back to the DMC) then I need to spend money from my } \\
\text { pocket for purchasing the envelope and paying the courier charge. (Participant in a } \\
\text { senior treatment supervisor FGD) }\end{array}$ \\
\hline
\end{tabular}

DMC, designated microscopy centre; FGD, focus group discussion; HCW, healthcare worker; PTLFU, pretreatment loss to follow-up; RNTCP, Revised National TB Control Programme; TB, tuberculosis. 
Table 5 Representative quotations on negative HCW attitudes and behaviours towards patients

\begin{tabular}{ll}
\hline HCW attitude or behaviour & Representative quotation \\
\hline Scolding patients & $\begin{array}{l}\text { Q32. The doctor scolded me, 'Why didn't you come at the time the infection started?' (Man } \\
\text { with a prior treatment history). }\end{array}$ \\
Stigmatising behaviour & $\begin{array}{l}\text { Q33. They (staff in the hospital) treated me in an irreverent way. (Family member of a man } \\
\text { without a prior treatment history) }\end{array}$
\end{tabular}

Q34. (D)octors don't have enough time to talk to patients. The staff nurse can counsel patients, but they treat the patients as untouchables. (Participant in a health visitor FGD).

$\begin{array}{ll}\begin{array}{l}\text { Violation of confidentiality or } \\ \text { disclosure of diagnosis }\end{array} & \begin{array}{l}\text { Q35. (When the TB diagnosis has been disclosed), patients will ask us, 'Why did you share } \\ \text { my information with the (community) leader?... Who gave you the right to share information } \\ \text { regarding my disease condition with others?... What will others think about me?' (Participant } \\ \text { in a health visitor FGD describing the type of language used by patients after disclosure of } \\ \text { diagnosis). }\end{array} \\ \text { Q36. Sometime we used to blackmail irregular patients by using permanent ID proof. For } \\ \begin{array}{l}\text { example, we got a driving license as proof of identification from an auto rickshaw driver } \\ \text { (patient). Then we said to him, 'If you take treatment irregularly, we will cancel your driving }\end{array} \\ \begin{array}{ll}\text { license.' (Participant in a senior treatment supervisor FGD). } \\ \text { care } \\ \text { Q37. Ward staff in the hospital were asking for bribes for towing patients to the X-ray } \\ \text { department. (Man with a prior treatment history). }\end{array} \\ \begin{array}{l}\text { Q38. We generally assign TB Numbers within a week or within } 2 \text { weeks. If the patient is regular } \\ \text { (on TB therapy), then we assign the TB Number the same day, but in the case of category II } \\ \text { patients (ie, those with a prior treatment history), we take two or more days for giving the TB } \\ \text { Number. (Participant in a senior treatment supervisor FGD). }\end{array} \\ \begin{array}{l}\text { Q39. They (nurses or supervising providers) do not want to care for the patient. For default } \\ \text { cases (ie, prior treatment history)... the nurse will say, 'Send him out. Don't give him a strip of } \\ \text { tablet.' Or, 'Why are you giving him tablets?' (Participant in a health visitor FGD describing the } \\ \text { type of language used by some HCWs towards some patients). }\end{array}\end{array}$

FGD, focus group discussion; HCW, healthcare worker; TB, tuberculosis.

system, because Laboratory Technicians, Health Visitors and Senior Treatment Supervisors fall under doctors' and nurses' supervision (Q29). Supervisors pulled HCWs away from data entry and patient interactions to perform tasks unrelated to their job taking care of TB patients:

I can't go and talk to the ward patient when he is available, because, at the same time, I will be called by the medical officer to do other work... I can't tell the doctors about the challenges I face. (Participant in a Health Visitor FGD).

HCWs lacked material support (eg, spending reimbursement) for tracking patients whose homes were inaccessible by public transportation $(\mathrm{Q} 30)$ and communicating with patients or other HCWs by phone:

For phone calls (with patients or other HCWs) we are spending almost 1000 rupees per month from our own pocket. (Participant in a Senior Treatment Supervisor FGD).

Resource constraints hindered coordination of patient referrals, since HCWs did not have money for phone or mail communication (Q31).

Thematic category 5: negative HCW attitudes and behaviours towards patients

Examples of negative or judgemental HCW attitudes and behaviours towards patients emerged in both patient and HCW data (table 5). HCWs scolded patients (Q32), especially those with a prior TB history, for whom challenges during previous treatment were perceived to have been due to moral shortcomings:

I told (the doctor) that my health condition improved quickly during my first TB treatment, which is why I had stopped therapy early. He said, 'Because of your disobedience in following your last treatment instructions, you are now suffering again'. (Man with a prior treatment history).

Some HCW conversations were perceived to be stigmatising by patients and HCWs (Q33 to Q34), sometimes including advice conveyed by providers:

Doctors will come and say, 'Close your mouth.' He asked me to maintain distance from my mother. How can I maintain distance from her? (Family member of a woman with a prior treatment history).

HCWs would sometimes notify community leaders or self-help group members (for female patients) about a patient's diagnosis with the goal of encouraging social support; however, HCWs themselves described how such actions sometimes irreparably harmed relationships with patients, who perceived these behaviours as violating their autonomy (Q35). Coercive behaviour aimed at encouraging treatment adherence sometimes resulted in poorer patient engagement in care (Q36). Patients described other negative HCW behaviours, such as requesting bribes to complete key steps in care (Q37). 
This spectrum of HCW behaviours included delaying registration for patients perceived to be at high risk for poor outcomes (Q38), as described in a Senior Treatment Supervisor FGD:

Senior Treatment Supervisor 5: In the initial days, patients take treatment with full enthusiasm, but later some patients stop coming back, so we keep their treatment cards separately...

Senior Treatment Supervisor 4: (A) few patients don't return, so we can't do anything more (to retrieve them)...

Moderator: So you never register that case?

Senior Treatment Supervisor 5: Yes, we never register that case. (Excerpt from a Senior Treatment Supervisor FGD)

Such behaviour not only raises concerns about transparency but also shapes patient perceptions that HCWs may not be invested in their care, as we will describe later. In rare cases, HCWs described other providers refusing care to patients perceived as 'irregular' or 'disobedient' (Q39), a finding echoed in patient experiences, as we will describe later.

\section{Towards an explanatory model for pretreatment loss to follow-up}

Through the mapping exercise, we identified interconnections among four of the thematic categories $(2,3,4$ and 5) that suggest a broader explanatory model for the health system's contribution to PTLFU. In particular, we identified two pathways leading to PTLFU that illuminate

\section{Pathway 1}

\begin{tabular}{|c|}
\hline $\begin{array}{l}\text { Constraints faced by healthcare workers } \\
\text { - Lack of material support to track patients } \\
\text { - Lack of material support to coordinate patient referrals } \\
\text { - High patient volume } \\
\text { - Hierarchy } \\
\text { Having to work at multiple facilities }\end{array}$ \\
\hline $\begin{array}{l}\text { Organizational and administrative barriers } \\
\text { faced by patients } \\
\text { - Challenges navigating within or between health facilities } \\
\text { - Not being notified of TB diagnosis } \\
\text { - Not being given general TB information } \\
\text { - Not progressing along care pathway due to healthcare } \\
\text { worker absenteeism, demand to present documents, or } \\
\text { infrastructure failures }\end{array}$ \\
\hline
\end{tabular}

Patients disengage from care due to frustration or resignation diverse ways that HCW constraints, attitudes and behaviours (thematic categories 4 and 5) shape patient experiences, attitudes and behaviours (thematic categories 2 and 3), ultimately leading to PTLFU (figure 2). We elucidate this explanatory model in more detail for each pathway.

\section{Pathway 1: "We just gave up and returned home."}

In the first pathway, social and resource constraints described by HCWs (thematic category 4) directly shaped patient experiences of health system organisational and administrative barriers (thematic category 2) (figure 2). For example, lack of material support for out-of-pocket costs resulted in HCWs being hesitant to make phone calls, mail patient-related documents or travel to patients' homes-actions that are critical to inform patients of their TB diagnoses, track patients' progress in reaching subsequent health facilities or inform other facilities to expect patients who had been referred. Such limitations contributed to patients' challenges in learning of their TB diagnoses or navigating between facilities.

High patient volume resulted in HCWs having limited time to counsel or accurately record patients' contact information, which contributed to patients' experiences of not being told their diagnoses or important general information about TB. Similarly, in the context of health system hierarchy, supervisors frequently pulled HCWs away from patient interactions, cutting short HCWs' opportunities to convey important information. Patients

\section{Pathway 2}
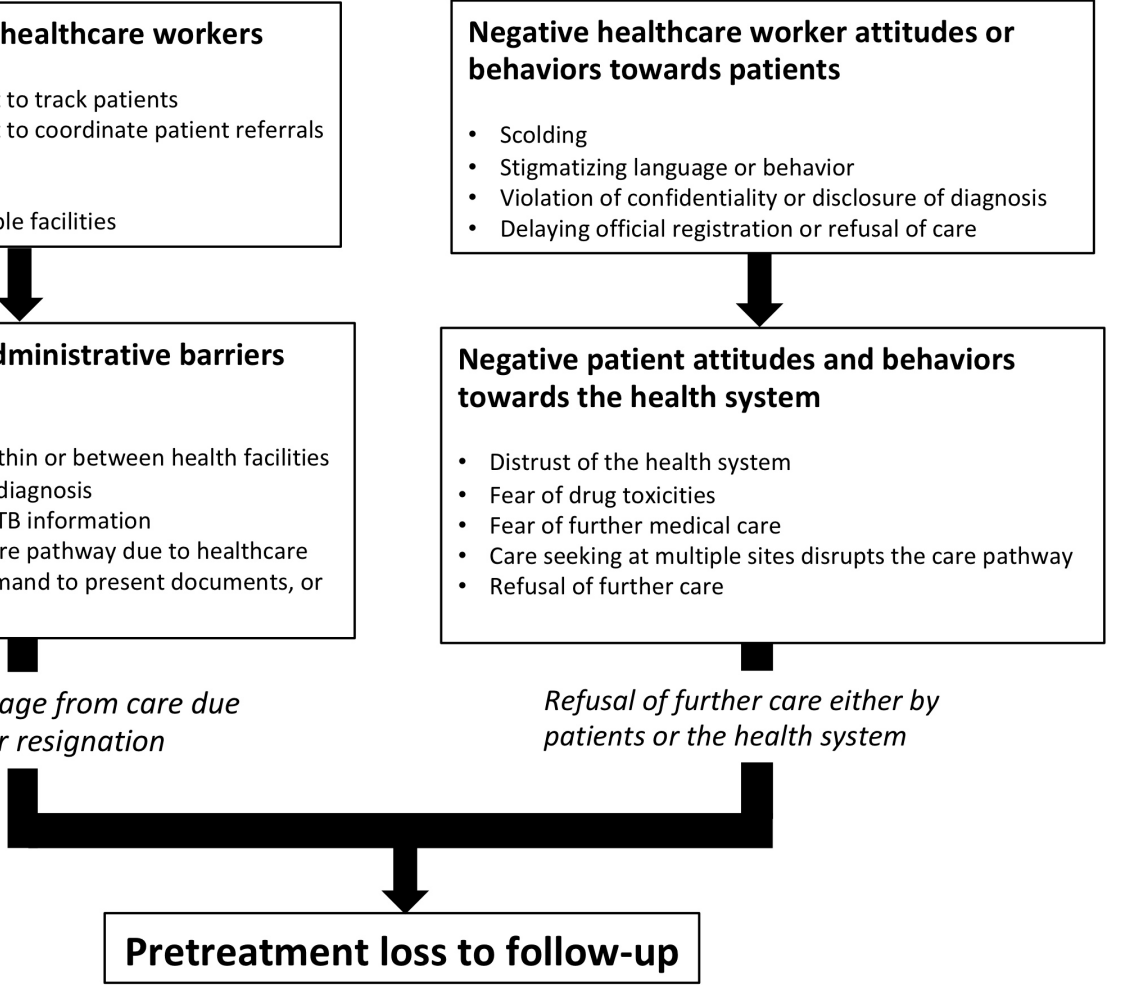

Figure 2 An explanatory model for the health system's role in contributing to pretreatment loss to follow-up of tuberculosis (TB) patients in Chennai, India. 
also reported showing up to health facilities for the next step in care (eg, picking up a test result, starting therapy), only to find that the relevant HCW was not available. From HCWs' perspectives, the reason for this problem was clear: they were responsible for providing care at multiple health facilities, such that they were sometimes only able to return to a given facility once a week.

When viewed in isolation, the organisational and administrative barriers to care experienced by patients in thematic category 2 paint a picture of them struggling to navigate a chaotic, intimidating and confusing health system. When contextualised within the social and resource constraints faced by HCWs, reasons for these barriers become more understandable.

Many patients who experienced PTLFU in this pathway did not have a prior treatment history (and therefore had less experience with the city TB programme), were initially referred for outpatient management (and therefore had to navigate to DOT centres to start treatment) and lived out of the city (and therefore often had to navigate to rural TB facilities for treatment). When evaluating individual narratives, it was evident that most patients experienced multiple barriers while navigating the care pathway. The effect of these hurdles was cumulative, provoking feelings of exhaustion, frustration and resignation that led patients to passively disengage from care despite having the intent and desire to get treatedas opposed to actively refusing care. One patient's family characteristically described this sense of resignation as follows:

After running from pillar to post, we just gave up and returned home. (Family member of a man without a prior treatment history).

\section{Pathway 2: "So I did not continue treatment."}

In the second pathway, negative or judgemental HCW attitudes and behaviours towards patients (thematic category 5) shaped negative patient attitudes and behaviours towards the health system (thematic category 3 ), resulting in refusal of care by either the patient or the health system (figure 2). Some of these HCW behaviours-including scolding or coercion-had the perceived goal of motivating or attempting to reengage patients perceived to be particularly challenging (table 2, Q35 above). However, such behaviours only increased patient distrust and fear of the health system:

Some of (the HCWs) talked nicely to me, but the doctor scolded me, so I didn't return to pick up my test result.

(Woman without a prior treatment history).

As another well-intentioned strategy, HCWs conducted home visits or involved community members in patients' care, such as local leaders or women's self-help group members. However, such involvement was often initiated without seeking permission and was perceived by patients as violating their autonomy, resulting in greater fear of the health system. HCWs themselves provided selfcritiques of these actions:
We once visited one of the (PTLFU) patients (at home) with our team, including the doctor, Senior Treatment Supervisor, Senior TB Laboratory Supervisor and Health Visitor. But he said 'I feel ashamed because of your action, so I cannot take medicines.' (Participant in a Health Visitor FGD)

In other situations, HCW behaviours reflected judgemental attitudes towards perceived moral failures of patients they deemed 'disobedient' or 'irregular' with therapy. These patients were presumed to be at higher risk for poor outcomes. HCWs sometimes delayed officially registering such patients to see if they would return to the clinic regularly (table 5, Q38). Patients were sometimes aware they had not been formally registered, which created a sense of being devalued by the health system:

They opened a treatment card for me, but they didn't give me a TB Number because they said I had been taking treatment irregularly. (Man with a prior treatment history)

In rare cases, HCWs were encouraged to refuse care to patients by their superiors, a situation described both by HCWs (table 5, Q39) and patients:

I went (to the treatment centre) but the Sister (nurse) said, 'Come back tomorrow.' She refused to provide me with any tablets so I became very vexed. I thought, 'Why did I come all the way here?' So I did not continue treatment. (Man with a prior treatment history).

Active refusal of further care in this pathway-whether by the health system or patients-is in contrast to the first pathway, in which patients disengaged from care due to frustration despite desiring to continue in care. Many patients who experienced PTLFU in the second pathway had a prior TB treatment history and advanced illness at presentation, such that they were more likely to be referred for inpatient admission and more likely to die before official registration. These characteristics shaped the attitudes and behaviours of both HCWs and patients. HCWs were more likely to view these patients as being high-risk and patients were more likely to distrust and fear the health system due to previous interactions with the city TB programme. As such, paradoxically, HCWs' assumptions - that these patients were 'disobedient' and 'irregular' - may have engendered these very behaviours from patients.

\section{DISCUSSION}

In this qualitative study of TB patients and HCWs in one of India's largest cities, our explanatory model highlights the critical role of the health system in contributing to PTLFU. We identified two health system pathways leading to poor outcomes, each with distinct implications for developing interventions to address this gap in care. In the first pathway, patients faced health system-related organisational and administrative barriers, which cumulatively resulted in disengagement from care due to frustration or resignation. By also capturing HCWs' perspectives, we were able to contextualise these health system 
barriers to show that they were not simply due to ineptitude but rather the result of deficits in human resources or material support HCWs needed to effectively do their jobs.

For example, difficulties in navigating within and between health facilities-especially DMCs where patients got diagnosed and DOT centres where treatment was initiated-was the most common barrier reported by patients. Numerous studies have highlighted circuitous pathways that patients traverse before TB diagnosis and treatment initiation in India, ${ }^{24-27}$ especially for those who initially seek private sector care. ${ }^{24}{ }^{26}$ Our study reveals similarly circuitous pathways within public sector care that contributed to patients not starting treatment, despite getting diagnosed. Patients were often not provided with the required information to make it to a DOT centre. Some patients in our sample had not returned to find out their sputum microscopy result and had not been notified of the result by phone.

From the HCW perspective, patients' navigational challenges were the result of constraints HCWs faced, such as lack of material support for making phone calls to patients or HCWs at other facilities. These constraints prevented HCWs from notifying patients of their TB diagnoses or coordinating referrals between facilities. HCWs also did not have administrative tools, such as information on DOT centre addresses, to provide patients with accurate referral information. Navigational challenges could potentially be addressed through interventions to improve communication and coordination, including short messaging service (SMS) notifications of test results to patients, ${ }^{28} 29$ electronic health records with real-time availability of patient information across facilities, ${ }^{15} 29$ paper-based or electronic directories with instructions to facilitate referral to other facilities or employment of patient navigators (individuals tasked with helping patients traverse the health system). ${ }^{30}$ Similarly, other barriers in pathway 1 are potentially rectifiable through practical health system changes, including hiring of more personnel, providing greater material support to HCWs (for phone calls, etc) and reducing administrative hurdles for patients.

The second pathway suggests that negative HCW attitudes regarding patients provoked patient attitudes of distrust, resulting in refusal of further care by the patient or the health system. HCWs conducted some activities with good intentions, such as home visits or disclosing a patient's diagnosis to community leaders; however, the approach to implementing such activities was often perceived by patients to be a violation of autonomy. In other cases, HCWs labelled patients as being 'disobedient' or 'irregular', judgements that resulted in negative consequences for patients, such as delayed registration or frank refusal of care by the health system. Even when HCWs did not refuse care to such patients, patients felt devalued, sometimes resulting in their own refusal of further care.
Our quantitative study showed that previously treated patients have a higher PTLFU rate than new TB patients, ${ }^{13}$ which is of concern as previously treated patients are more likely to have drug-resistant $\mathrm{TB} .{ }^{31}$ In the current qualitative study, negative HCW attitudes and behaviours of delayed registration and refusal of care were particularly common for previously treated patients. As also shown in a previous Indian study, ${ }^{7}$ patients with a prior TB treatment history also displayed greater distrust of the health system resulting from their prior experiences with the TB programme, including negative interactions with HCWs, challenges engaging with facility-based DOT and adverse effects of TB medications. Whether intentional or not, delayed registration of these patients may result in the appearance of more favourable programmatic outcomes (eg, higher rates of treatment completion). ${ }^{13}$ Such actions not only contribute to the health system's lack of transparency and accountability, but these actions may also exclude socially marginalised patients from care. $^{32}$

A key implication of this second pathway is that, because tensions lie within the HCW-patient interaction, sensitising HCWs to transform their attitudes and behaviours may be required. In addition, tensions in patients' interactions with HCWs could continue to contribute to loss to follow-up during the TB treatment course, as has been suggested in prior Indian ${ }^{33} 34$ and global $^{22}$ literature. Our findings support the importance of measuring and improving patients' user experience of the health system. ${ }^{35}$ Assessing user experience through patient surveys and incentivising HCWs who receive good reports may also improve the HCW-patient interaction. Our findings are consistent with previous studies revealing patient hardship due to India's facility-based DOT model, ${ }^{34}$ although use of digital adherence technologies is facilitating self-administered therapy for patients in some parts of the country. ${ }^{3637}$

Although our explanatory model focusses on health system barriers, our intention is not to minimise patientrelated, family-related and society-related barriers contributing to PTLFU (thematic category 1). Alcohol use was particularly common and has been previously described as a barrier to TB care in Chennai. ${ }^{38}$ Interventions to address alcohol use have been associated with improvements in treatment success. ${ }^{39}$ Family-related and societyrelated barriers also included lack of social support and TB-related stigma. Some patients had advanced disease at the time of diagnosis, making it more difficult to navigate the health system and resulting in patients dying before official registration. This highlights a need to diagnose patients earlier in the disease course, by reducing diagnostic delays through active case-finding initiatives ${ }^{40} 41$ and better training of HCWs, who frequently miss patients presenting with early TB symptoms. ${ }^{4243}$ Challenges at the level of patients, their families or society were amplified by the health system barriers described in our explanatory model. For example, alcohol use disorder contributed to HCWs' moral judgements of patients being 'disobedient', 
and advanced illness accentuated the already considerable challenges of health system navigation.

Strengths of our study include its in-depth analysis of narrative data from patients and HCWs to assemble a preliminary explanatory model to guide interventions for health system barriers contributing to PTLFU. In addition, qualitative data collection was embedded within a quantitative cohort study, allowing us to achieve a more nuanced understanding of PTLFU than is possible using either approach alone. For example, after finding that previously treated patients have greater risk of PTLFU, ${ }^{13}$ we delved into unique challenges faced by these patients in the qualitative study.

A study limitation is that more than one-third of PTLFU patients were untrackable due to missing contact information in government records, and a few patients declined to participate in qualitative interviews. While we cannot be sure how the absence of these patients biassed our findings, it is possible that some of these patients represent individuals with greater distrust of the health system, as implied from their hesitation to engage with HCWs and our research team. It is also possible that some untrackable patients represent individuals with greater mobility (eg, truck drivers, migrant labourers) or who temporarily visited Chennai from nearby districts to access medical care. ${ }^{15}$ Another study limitation is the small number of women in our sample, which limits our ability to draw conclusions regarding how sex influences PTLFU. Future studies may consider enriching the number of women in their sample to better understand women's perspectives and potential differences in reasons for PTLFU by sex.

\section{CONCLUSIONS}

In this qualitative study, we found that PTLFU is a complex problem involving diverse reasons that patients do not start treatment or get officially registered in the TB programme. A unifying finding of our explanatory model was the prominent role of health system barriers in contributing to PTLFU for most patients-directly and by amplifying patient-related challenges to engaging in care. Health system barriers play a particularly prominent role for previously treated patients, who are at higher risk for having drug-resistant TB. Assumptions that some TB patients are 'disobedient' or 'irregular' may influence HCW attitudes and behaviours in a manner that paradoxically engenders such behaviours from these patients, resulting in PTLFU and other poor outcomes.

Addressing this gap in $\mathrm{TB}$ care delivery will require development of theory-informed multifaceted interventions that address organisational and administrative health system barriers and transform HCW attitudes towards patients. ${ }^{44}$ Such interventions should focus on making it easier for patients to navigate the health system, ensuring prompt communication of diagnoses to patients, reducing constraints that prevent HCWs from effectively performing their jobs and educating HCWs in non-judgemental approaches to care. These strategies have potential to reduce PTLFU while ensuring patientcentred care to all individuals living with $\mathrm{TB}$, as envisioned by the WHO's End TB Strategy. ${ }^{45}$

\section{Author affiliations}

${ }^{1}$ Department of Social and Behavioral Research, National Institute for Research in Tuberculosis, Chennai, Tamil Nadu, India

${ }^{2}$ District TB Office, Chennai, Tamil Nadu, India

${ }^{3}$ Department of Public Health and Community Medicine and Center for Global Public Health, Tufts University School of Medicine, Boston, Massachusetts, USA

${ }^{4}$ Fenway Institute, Boston, Massachusetts, USA

${ }^{5}$ Division of Infectious Diseases, Beth Israel Deaconess Medical Center and Harvard Medical School, Boston, Massachusetts, USA

${ }^{6}$ World Health Organization Headquarters, Geneva, Switzerland

${ }^{7}$ Division of Geographic Medicine and Infectious Diseases, Tufts Medical Center, Boston, Massachusetts, USA

Contributors BET led project management and supervision of field data collection, and she supported study conceptualisation, design and data interpretation. S Sellappan contributed to study design, project management and field data collection. CS contributed to project management and field data collection. $\mathrm{JL}$ contributed to study conceptualisation, study design and facilitated field data collection. SL contributed to field data collection and data entry. AR contributed to field data collection and data entry. BJ contributed to field data collection and data entry. SK contributed to field data collection and data entry. SA contributed to field data collection and data entry. ML contributed to data analysis, data interpretation and initial drafting of the manuscript. AG contributed to data analysis, data interpretation and initial drafting of the manuscript. KM contributed to study conceptualisation, study design, acquisition of funding and data interpretation. S Swaminathan contributed to study conceptualisation, study design and data interpretation. RS led study conceptualisation, study design, acquisition of funding, data analysis, data interpretation and initial drafting of the manuscript. RS also contributed to data entry, project management and supervision of field data collection. All authors provided critical revisions to the initial manuscript draft and approved the final paper.

Funding RS and the field research team were supported by a Fogarty Global Health Equity Scholars Fellowship (NIAID R25 TW009338). RS also received support from a Harvard Catalyst KL2/Catalyst Medical Investigator Training Award (KL2 TR001100) and a Doris Duke Clinical Scientist Development Award. The funding bodies had no role in study design, data collection, data analysis, data interpretation or manuscript writing.

Competing interests None declared.

Patient and public involvement Patients and/or the public were involved in the design, or conduct, or reporting or dissemination plans of this research. Refer to the Methods section for further details.

\section{Patient consent for publication Not required.}

Ethics approval The research protocol for this study was approved by the Institutional Ethics Committee of the National Institute for Research in TB (NIRT) (FWA00005104) on 29 December 2014 and the Institutional Review Board of Brigham and Women's Hospital (Partners Healthcare) (FWA00000484) on 13 January 2015. Written informed consent was collected from all PTLFU patients who agreed to participate in qualitative interviews. In addition to anonymising identifying patient information, in many cases we have anonymised names of health facilities if patient or family member comments might reflect poorly upon that facility.

Provenance and peer review Not commissioned; externally peer reviewed.

Data availability statement Data are available upon request. The qualitative data have not been included as a supplement because this would likely compromise the individual privacy of patients, as it may be possible to identify specific individuals based on the in-depth narratives. Requests for the de-identified qualitative data set can be made by contacting Dr Beena Thomas (beenathomas@nirt.res.in), although access to these data may be subject to Indian Council of Medical Research policies.

Open access This is an open access article distributed in accordance with the Creative Commons Attribution Non Commercial (CC BY-NC 4.0) license, which permits others to distribute, remix, adapt, build upon this work non-commercially, and license their derivative works on different terms, provided the original work is 
properly cited, appropriate credit is given, any changes made indicated, and the use is non-commercial. See: http://creativecommons.org/licenses/by-nc/4.0/.

\section{ORCID iD}

Ramnath Subbaraman http://orcid.org/0000-0002-2063-943X

\section{REFERENCES}

1 Subbaraman R, Nathavitharana RR, Mayer $\mathrm{KH}$, et al. Constructing care cascades for active tuberculosis: a strategy for program monitoring and identifying gaps in quality of care. PLoS Med 2019;16:e1002754.

2 MacPherson P, Houben RMGJ, Glynn JR, et al. Pre-Treatment loss to follow-up in tuberculosis patients in low- and lower-middleincome countries and high-burden countries: a systematic review and meta-analysis. Bull World Health Organ 2014;92:126-38.

3 Naidoo P, Theron G, Rangaka MX, et al. The South African tuberculosis care cascade: estimated losses and methodological challenges. J Infect Dis 2017;216:S702-13.

4 Subbaraman R, Nathavitharana RR, Satyanarayana S, et al. The tuberculosis cascade of care in India's public sector: a systematic review and meta-analysis. PLoS Med 2016;13:e1002149.

5 Dandona R, Dandona L, Mishra A, et al. Utilization of and barriers to public sector tuberculosis services in India. Natl Med J India 2004;17:292-9.

6 Gopi PG, Chandrasekaran V, Subramani R, et al. Failure to initiate treatment for tuberculosis patients diagnosed in a community survey and at health facilities under a dots program in a district of South India. Indian J Tuberc 2005;52:153-6.

7 Mehra D, Kaushik RM, Kaushik R, et al. Initial default among sputum-positive pulmonary TB patients at a referral hospital in Uttarakhand, India. Trans R Soc Trop Med Hyg 2013;107:558-65.

8 Pillai D, Purty A, Prabakaran S, et al. Initial default among tuberculosis patients diagnosed in selected medical colleges of Puducherry: issues and possible interventions. Int J Med Sci Public Health 2015;4:957-4.

9 Mandal A, Basu M, Das P, et al. Magnitude and reasons of initial default among new sputum positive cases of pulmonary tuberculosis under RNTCP in a district of West Bengal, India. SE Asia J Pub. Health 2015;4:41-7.

10 Tripathy JP, Srinath S, Naidoo P, et al. Is physical access an impediment to tuberculosis diagnosis and treatment? A study from a rural district in North India. Public Health Action 2013;3:235-9.

11 Dave P, Nimavat P, Shah A, et al. Knowing more about initial default among diagnosed sputum smear-positive pulmonary tuberculosis patients in Gujarat, India [Abstract PC-868-03]. Int J Tuberc Lung Dis 2013;17:S469

12 Sai Babu B, Satyanarayana AVV, Venkateshwaralu G, et al. Initial default among diagnosed sputum smear-positive pulmonary tuberculosis patients in Andhra Pradesh, India. Int J Tuberc Lung Dis 2008;12:1055-8.

13 Thomas BE, Subbaraman R, Sellappan S, et al. Pretreatment loss to follow-up of tuberculosis patients in Chennai, India: a cohort study with implications for health systems strengthening. BMC Infect Dis 2018;18:142.

14 Dhanaraj B, Papanna MK, Adinarayanan S, et al. Prevalence and risk factors for adult pulmonary tuberculosis in a metropolitan city of South India. PLoS One 2015;10:e0124260.

15 Subbaraman R, Thomas BE, Sellappan S, et al. Tuberculosis patients in an Indian mega-city: where do they live and where are they diagnosed? PLoS One 2017;12:e0183240.

16 Creswell J, Klassen A, Clark V, et al. Best practices for mixed methods research in the health sciences. NIH Office of Behavioral and Social Sciences Research, 2011.

17 Creswell JW. Research design: qualitative, quantitative, and mixed methods approaches. Thousand Oaks, CA: Sage Publications, 2013.

18 Noblit G, Hare R. Meta-ethnography: synthesizing qualitative studies. Newbury Park. CA: Sage, 1988.

19 Thomas J, Harden A. Methods for the thematic synthesis of qualitative research in systematic reviews. BMC Med Res Methodol 2008;8:45

20 Ware NC, Wyatt MA, Geng EH, et al. Toward an understanding of disengagement from HIV treatment and care in sub-Saharan Africa: a qualitative study. PLoS Med 2013;10:e1001369.

21 Merten S, Kenter E, McKenzie O, et al. Patient-Reported barriers and drivers of adherence to antiretrovirals in sub-Saharan Africa: a meta-ethnography. Trop Med Int Health 2010;15:16-33.
22 Munro SA, Lewin SA, Smith HJ, et al. Patient adherence to tuberculosis treatment: a systematic review of qualitative research. PLoS Med 2007;4:e238.

23 Ahmed S, Autrey J, Katz IT, et al. Why do people living with HIV not initiate treatment? A systematic review of qualitative evidence from low- and middle-income countries. Soc Sci Med 2018;213:72-84.

24 Sreeramareddy CT, Qin ZZ, Satyanarayana S, et al. Delays in diagnosis and treatment of pulmonary tuberculosis in India: a systematic review. Int J Tuberc Lung Dis 2014;18:255-66.

25 Mistry N, Rangan S, Dholakia Y, et al. Durations and delays in care seeking, diagnosis and treatment initiation in uncomplicated pulmonary tuberculosis patients in Mumbai, India. PLoS One 2016;11:e0152287.

26 Veesa KS, John KR, Moonan PK, et al. Diagnostic pathways and direct medical costs incurred by new adult pulmonary tuberculosis patients prior to anti-tuberculosis treatment - Tamil Nadu, India. PLoS One 2018;13:e0191591.

27 Yellappa V, Lefèvre P, Battaglioli T, et al. Patients pathways to tuberculosis diagnosis and treatment in a fragmented health system: a qualitative study from a South Indian district. BMC Public Health 2017;17:635.

28 Wagstaff A, van Doorslaer E, Burger R. SMS nudges as a tool to reduce tuberculosis treatment delay and pretreatment loss to followup. A randomized controlled trial. PLoS One 2019;14:e0218527.

29 Mehta K, Kumar AMV, Chawla S, et al. 'M-TRACK' (mobile phone reminders and electronic tracking tool) cuts the risk of pre-treatment loss to follow-up by $80 \%$ among people living with HIV under programme settings: a mixed-methods study from Gujarat, India. Glob Health Action 2018;11:1438239.

30 McBrien KA, Ivers N, Barnieh L, et al. Patient navigators for people with chronic disease: a systematic review. PLoS One 2018;13:e0191980

31 Ministry of Health and Family Welfare. Report of the first national anti-tuberculosis drug resistance survey of India 2014-16. New Delhi, India: Ministry of Health and Family Welfare, 2018.

32 Singh V, Jaiswal A, Porter JDH, et al. TB control, poverty, and vulnerability in Delhi, India. Trop Med Int Health 2002;7:693-700.

33 Jaiswal A, Singh V, Ogden JA, et al. Adherence to tuberculosis treatment: lessons from the urban setting of Delhi, India. Trop Med Int Health 2003;8:625-33.

34 Yellappa V, Lefèvre P, Battaglioli T, et al. Coping with tuberculosis and directly observed treatment: a qualitative study among patients from South India. BMC Health Serv Res 2016;16:283.

35 Kruk ME, Gage AD, Arsenault C, et al. High-Quality health systems in the sustainable development goals era: time for a revolution. Lancet Glob Health 2018;6:e1196-252.

36 Cross A, Gupta N, Liu B, et al. 99DOTS: A low-cost approach to monitoring and improving medication adherence. ICTD '19. Ahmedabad, India, 2019.

37 Subbaraman R, de Mondesert L, Musiimenta A, et al. Digital adherence technologies for the management of tuberculosis therapy: mapping the landscape and research priorities. BMJ Glob Health 2018;3:e001018.

38 Suhadev M, Thomas BE, Raja Sakthivel M, et al. Alcohol use disorders (AUD) among tuberculosis patients: a study from Chennai, South India. PLoS One 2011;6:e19485.

39 Thomas B, Watson B, Senthil EK, et al. Alcohol intervention strategy among tuberculosis patients: a pilot study from South India. Int $J$ Tuberc Lung Dis 2017;21:947-52.

40 Prasad BM, Satyanarayana S, Chadha SS, et al. Experience of active tuberculosis case finding in nearly 5 million households in India. Public Health Action 2016;6:15-18.

41 Shewade HD, Gupta V, Satyanarayana S, et al. Patient characteristics, health seeking and delays among new sputum smear positive TB patients identified through active case finding when compared to passive case finding in India. PLoS One 2019;14:e0213345

42 Kwan A, Daniels B, Saria V, et al. Variations in the quality of tuberculosis care in urban India: a cross-sectional, standardized patient study in two cities. PLoS Med 2018;15:e1002653.

43 Satyanarayana S, Subbaraman R, Shete P, et al. Quality of tuberculosis care in India: a systematic review. Int J Tuberc Lung Dis 2015;19:751-63.

44 Chaisson LH, Katamba A, Haguma P, et al. Theory-Informed interventions to improve the quality of tuberculosis evaluation at Ugandan health centers: a quasi-experimental study. PLoS One 2015;10:e0132573

45 Uplekar M, Weil D, Lonnroth K, et al. Who's new end TB strategy. Lancet 2015;385:1799-801. 